\title{
UNIVERSAL TRUNCATION ERROR UPPER BOUNDS IN SAMPLING RESTORATION
}

\author{
ANDRIY YA. OLENKO AND TIBOR K. POGÁNY
}

\begin{abstract}
Universal (pointwise uniform and time shifted) truncation error upper bounds are presented for the Whittaker-Kotel'nikov-Shannon (WKS) sampling restoration sum for Bernstein function classes $B_{\pi, d}^{q}, q>1, d \in \mathbb{N}$, when the decay rate of the sampled functions is unknown. The case of regular sampling is discussed. Extremal properties of related series of sinc functions are investigated.
\end{abstract}

\section{Introduction}

Let $\mathrm{X}$ be a normed space and assume that the structure of $\mathrm{X}$ admits the sampling restoration procedure

$$
f(\mathbf{x})=\sum_{\mathbf{n} \in \mathbb{Z}^{d}} f\left(t_{\mathbf{n}}\right) S\left(\mathbf{x}, t_{\mathbf{n}}\right) \quad(f \in \mathbf{X})
$$

where $\mathbf{x} \in \mathbb{R}^{d}, \mathfrak{T}:=\left\{t_{\mathbf{n}}\right\}_{\mathbf{n} \in \mathbb{Z}^{d}} \subset \mathbb{R}^{d}$ is the set of sampling points and $S(\cdot, \cdot)$ is the sampling function. This formula is one of the basic tools in signal processing.

In direct numerical implementations we consider the truncated variant of (1), that is

$$
Y_{\mathfrak{J}}(f ; \mathbf{x})=\sum_{\mathbf{n} \in \mathfrak{J}} f\left(t_{\mathbf{n}}\right) S\left(\mathbf{x}, t_{\mathbf{n}}\right) \quad\left(\mathfrak{J} \subset \mathbb{Z}^{d}\right),
$$

where the index set $\mathfrak{J}$ is necessarily finite in applications. Namely, restoring the continuous signal from discrete samples or assessing the information lost in the sampling process are the fundamental problems in sampling and interpolation theory.

The usual procedure is to estimate the truncation error

$$
\left\|T_{\mathfrak{J}}(f ; \mathbf{x})\right\|:=\left\|f(\mathbf{x})-Y_{\mathfrak{J}}(f ; \mathbf{x})\right\| \leq \varphi_{\mathfrak{J}}(f ; \mathbf{x}),
$$

2000 Mathematics Subject Classification. Primary: 94A20, 41A25; Secondary: 41A05, 41A17, 41A80, 26D15.

Key words and phrases. Whittaker-Kotel'nikov-Shannon sampling restoration formula, approximation/interpolation error level, Plancherel-Pólya inequality, Bernstein function class, regular sampling theorem, truncation error upper bound, multidimensional sampling, sinc functions, incomplete Lambda function.

THIS IS AN AUTHOR'S ACCEPTED MANUSCRIPT OF AN ARTICLE PUBLISHED IN THE GEORGIAN MATHEMATICAL JOURNAL. VOL. 17, NO. 4. (2010), 765-786. THE FINAL PUBLICATION IS AVAILABLE AT DE GRUYTER. DOI: $10.1515 /$ GMJ.2010.033 
where $\|\cdot\|$ could denote any suitable norm and $\varphi_{\mathfrak{J}}$ denotes the truncation error upper bound. Simple truncation error upper bounds are the main tools in numerical implementations, when they do not contain infinite products and/or unknown function values. However, a sharp truncation error upper bound enables pointwise, almost sure, uniform etc. convergence of the approximating sequence $Y_{\mathfrak{J}}(f ; \mathbf{x})$ to the initial $f \in \mathbf{X}$ when $|\mathfrak{J}| \rightarrow \infty$.

In their recent article [1] the authors established sharp upper bounds for interpolation remainders of the multidimensional Paley-Wiener class functions in the $L^{2}$-space, in the case of finite, regular (equidistantly sampled) WKS sampling sums and consequent extremal functions are given [1, Theorems 1, 2]. Also, truncation error analysis and convergence rate is provided in weak Cramér class random fields [1, Theorems 3, 4]. Here we generalize in some fashion certain our findings to Bernstein function class in the $L^{p}$-spaces.

The main aim of this paper is to discuss $T_{\mathfrak{J}}(f ; \mathbf{x})$ when $f \in L^{p}$ is coordinatewise exponentially bounded entire function, that is, belongs to suitable Bernstein functions class, in different situations by obtaining pointwise upper bounds valid for all $\mathrm{x}$ belonging to the signal domain [1]. We call this kind of upper bound universal.

All numerical computations in the paper were done using Mathematica 5.0 .

\section{Multidimensional Plancherel-Pólya inequality}

The following few results will prove useful throughout. The first one is the multidimensional analogue of the famous Plancherel-Pólya inequality extended from the one-dimensional case published in [2, 3].

Denote $\|\cdot\|_{p}$ the $L_{p}$-norm (while $\|\cdot\|_{\infty} \equiv \operatorname{ess} \sup |\cdot|$ ) and let $L^{p}(\mathbb{R})$ be the class of all complex-valued functions whose restrictions to $\mathbb{R}$ have finite $L_{p}$-norm. Assume $f \in L^{r}(\mathbb{R}), r>0$ to be of exponential type $\sigma>0$ and let $\left\{t_{n}\right\}_{n \in \mathbb{Z}}$ be a separated real sequence, i.e. such that $\inf _{n \neq m}\left|t_{n}-t_{m}\right| \geq \delta>0$. Then we have [3, Eq. (76)]

$$
\sum_{n \in \mathbb{Z}}\left|f\left(t_{n}\right)\right|^{r} \leq B\|f\|_{r}^{r},
$$

where

$$
B=\frac{8\left(e^{r \delta \sigma / 2}-1\right)}{r \pi \sigma \delta^{2}} .
$$

Formula (3) is the celebrated Plancherel-Pólya inequality. It could be mentioned that Boas [4] established another estimate in the one-dimensional case under different assumptions on $\mathfrak{T}$ and recently Lindner published an estimate in the one-dimensional case when $p=2,[5]$.

Now, we give the multidimensional analogue of the Plancherel-Pólya inequality. Here, and in what follows $B_{\sigma, d}^{r}, r>0$ denotes the Bernstein class [6] of $d$-variable entire functions of exponential type at most $\boldsymbol{\sigma}=\left(\sigma_{1}, \cdots, \sigma_{d}\right)$ coordinatewise whose restriction to $\mathbb{R}^{d}$ is in $L^{r}\left(\mathbb{R}^{d}\right)$. 
We will use the following Nikolskiı-type inequality of different dimensions.

Lemma 1. [6, §3.4.2] If $1 \leq r \leq \infty$ and $1 \leq m \leq d$, then for each entire function $g(\mathbf{z}) \in L^{r}\left(\mathbb{R}^{d}\right)$ of exponential type $\boldsymbol{\sigma}$ the following inequality holds

$$
\begin{aligned}
& \left(\int_{\mathbb{R}^{m}}\left|g\left(z_{1}, \cdots, z_{m}, z_{m+1}, \cdots, z_{d}\right)\right|^{r} \mathrm{~d} z_{1} \cdots \mathrm{d} z_{m}\right)^{1 / r} \\
& \quad \leq 2^{d-m}\left(\prod_{k=m+1}^{d} \sigma_{k}\right)^{1 / r}\left(\int_{\mathbb{R}^{d}}\left|g\left(z_{1}, \cdots, z_{d}\right)\right|^{r} \mathrm{~d} z_{1} \cdots \mathrm{d} z_{d}\right)^{1 / r} .
\end{aligned}
$$

For fixed $d$ and $m$ and arbitrary $\boldsymbol{\sigma}=\left(\sigma_{1}, \cdots, \sigma_{d}\right)$ this inequality is exact in the sense of order.

Theorem 1. Let $f \in B_{\boldsymbol{\sigma}, d}^{r}, r \geq 1$ and let $\mathfrak{T}=\left\{t_{\mathbf{n}}=\left(t_{n_{1}}, \ldots t_{n_{d}}\right)\right\}_{\mathbf{n} \in \mathbb{Z}^{d}}$, be real separated sequence, that is

$$
\inf _{n_{\ell} \neq m_{\ell}}\left|t_{n_{\ell}}-t_{m_{\ell}}\right| \geq \delta_{\ell}>0 \quad(\ell=1, \cdots, d) .
$$

Then

$$
\sum_{\mathbf{n} \in \mathbb{Z}^{d}}\left|f\left(t_{\mathbf{n}}\right)\right|^{r} \leq \mathfrak{B}_{d, r}\|f\|_{r}^{r},
$$

where

$$
\mathfrak{B}_{d, r}=\left(\frac{8}{r \pi}\right)^{d} \prod_{\ell=1}^{d} \frac{e^{r \delta_{\ell} \sigma_{\ell} / 2}-1}{\sigma_{\ell} \delta_{\ell}^{2}}
$$

Proof. Take $d=2$; the proof will be identical in the case $d>2$. By assumption $f \in B_{\boldsymbol{\sigma}, d}^{r}$ and bearing in mind Lemma 1 1 which holds for $r \geq 1$, we conclude that $f\left(\cdot, x_{2}\right) \in B_{\sigma_{1}, 1}^{r}$ and $f\left(x_{1}, \cdot\right) \in B_{\sigma_{2}, 1}^{r}$. Therefore, we can apply (3) coordinatewise to $\sum_{\mathbf{n} \in \mathbb{Z}^{d}}\left|f\left(t_{\mathbf{n}}\right)\right|^{r}$. Because $\left\{t_{n_{\ell}}\right\}_{n_{\ell} \in \mathbb{Z}}$ are separated with $\delta_{\ell}, \ell=1,2$ we deduce

$$
\begin{aligned}
\sum_{\mathbf{n} \in \mathbb{Z}^{2}}\left|f\left(t_{\mathbf{n}}\right)\right|^{r} & =\sum_{n_{1} \in \mathbb{Z}}\left(\sum_{n_{2} \in \mathbb{Z}}\left|f\left(t_{n_{1}}, t_{n_{2}}\right)\right|^{r}\right) \\
& \leq \frac{8\left(e^{r \delta_{2} \sigma_{2} / 2}-1\right)}{r \pi \sigma_{2} \delta_{2}^{2}} \sum_{n_{1} \in \mathbb{Z}} \int_{\mathbb{R}}\left|f\left(t_{n_{1}}, x_{2}\right)\right|^{r} \mathrm{~d} x_{2} \\
& =\frac{8\left(e^{r \delta_{2} \sigma_{2} / 2}-1\right)}{r \pi \sigma_{2} \delta_{2}^{2}} \int_{\mathbb{R}}\left(\sum_{n_{1} \in \mathbb{Z}}\left|f\left(t_{n_{1}}, x_{2}\right)\right|^{r}\right) \mathrm{d} x_{2} .
\end{aligned}
$$

The second subsequent application of (3) to (6) yields

$$
\sum_{\mathbf{n} \in \mathbb{Z}^{2}}\left|f\left(t_{\mathbf{n}}\right)\right|^{r} \leq \frac{64\left(e^{r \delta_{1} \sigma_{1} / 2}-1\right)\left(e^{r \delta_{2} \sigma_{2} / 2}-1\right)}{\sigma_{1} \sigma_{2} r^{2} \pi^{2}\left(\delta_{1} \delta_{2}\right)^{2}} \int_{\mathbb{R}^{2}}\left|f\left(x_{1}, x_{2}\right)\right|^{r} \mathrm{~d} x_{1} \mathrm{~d} x_{2}=\mathfrak{B}_{2, r}\|f\|_{r}^{r},
$$

where in (77) Fubini's theorem is used. So, the assertion of the theorem is proved. 
Remark 1. In [3, §46, p.148] the authors assume the condition

$$
\sum_{\ell=1}^{d}\left|t_{\mathbf{n}}^{(\ell)}-t_{\mathbf{m}}^{(\ell)}\right|^{2} \geq \Delta^{2}
$$

on $\mathfrak{T}$ and $p>0$, so that the multidimensional variant of (3) holds. It is clear that our separability assumption upon $\mathfrak{T}$ is stronger then (8), where

$$
\Delta^{2}=\sum_{\ell=1}^{d} \delta_{\ell}^{2}
$$

could be taken. Our approach results in the explicit Plancherel-Pólya-type constant (5) such that plays crucial role in establishing explicit (not necessarily exact) truncation error upper bounds in the multidimensional WhittakerKotel'nikov-Shannon (WKS) sampling theorem, being a part of numerical routines in computation.

Multidimensional Plancherel-Pólya inequalities can be found e.g. in Triebel's book [7]; also, during last years several additional very far going generalizations of the multidimensional Plancherel-Pólya inequality were obtained, among others in [8], [9]. Unfortunately, no explicit estimates of the Plancherel-Pólya constant have appeared neither in these articles, nor in articles referenced therein including the 'ancestor' article [3].

\section{On extrema of $\sum|\operatorname{sinc}(x-n)|^{p}$ and their properties}

Another frequently needed mathematical tool concerns upper bound estimates for $\sum|\operatorname{sinc}(x-n)|^{p}, p>1$. Here $\operatorname{sinc}(x):=\frac{\sin (\pi x)}{\pi x}$ for $x \neq 0$ and $\operatorname{sinc}(0):=1$.

Theorem 2. There holds

$$
\sum_{n \in \mathbb{Z}}|\operatorname{sinc}(x-n)|^{p} \leq \mathfrak{C}_{p} \quad(x \in \mathbb{R})
$$

where

$$
\mathfrak{C}_{p}:=\left\{\begin{array}{ll}
1+\left(\frac{2}{\pi}\right)^{p} \frac{p}{p-1} & 1<p<2 \\
1 & p \geq 2
\end{array} .\right.
$$

The statement is the straightforward consequence of lemmata [10, Lemma 11.2] and [11, Lemma 2.4], so we omit the proof.

Let $\mathfrak{J}=\mathfrak{J}_{x}:=\{n:|x-n| \leq N\}, x \in \mathbb{R}, N \in \mathbb{N}$; accordingly let us define

$$
\mathfrak{h}_{p, N}(x):=\sum_{n \notin \mathfrak{J}_{x}}|\operatorname{sinc}(x-n)|^{p} .
$$

It was shown [12] that for arbitrary $N \in \mathbb{N}$ the function $\max \mathfrak{h}_{2, N}(x)=\mathfrak{h}_{2, N}(1 / 2)$. We begin by showing that this is not true for $p$ and $N$ in general - compare the Figures 1,2 below. 


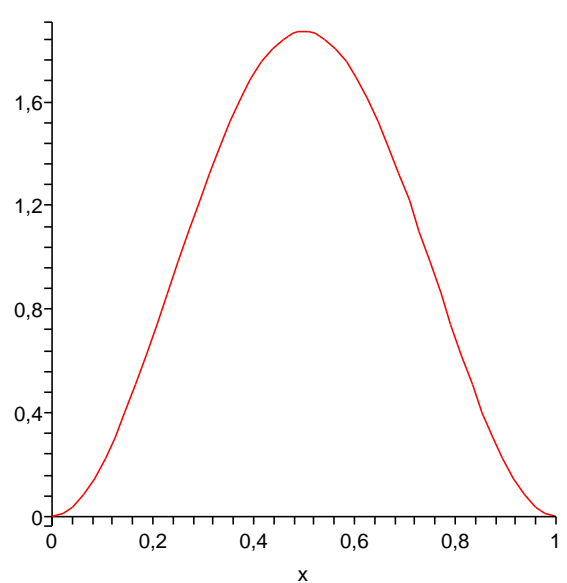

Figure $1 . \mathfrak{h}_{2,2}(x)$

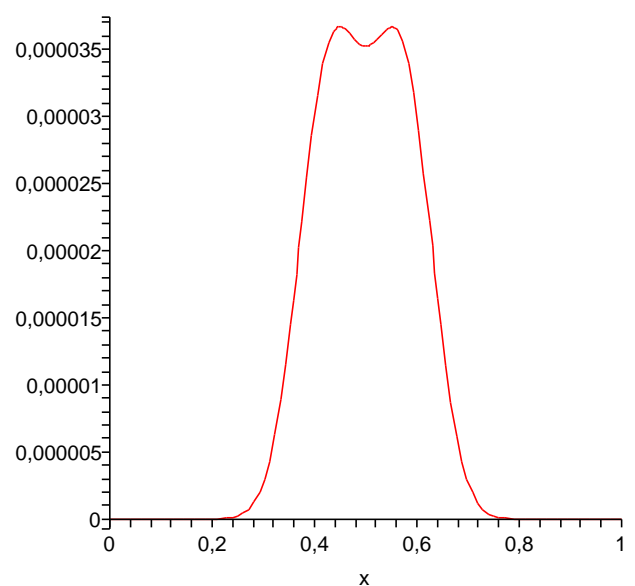

Figure 2. $\mathfrak{h}_{27,2}(x)$

Let us note that $\mathfrak{h}_{p, N}(x)$ is 1-periodic; furthermore it is symmetric with respect to $x=1 / 2$ and $\mathfrak{h}_{p, N}(1):=0$. Therefore, it is enough to study this sum only on the interval $[1 / 2,1)$. However, equivalently

$$
\mathfrak{h}_{p, N}(x)=\sin ^{p}(\pi x) \sum_{k=N+1}^{\infty}\left(\frac{1}{(k-x)^{p}}+\frac{1}{(k+x-1)^{p}}\right) .
$$

One introduces the incomplete Lambda function $\lambda(s ; a)$ defined by the series

$$
\lambda(s ; a)=\sum_{n=1}^{\infty} \frac{1}{(2(n+a)-1)^{s}} \quad(s>1, a \geq 0) .
$$

Theorem 3. For each $N$ there exists $p_{*}$ such that $\left(1 / 2, \mathfrak{h}_{p, N}(1 / 2)\right)$ is a local minimum of $\mathfrak{h}_{p, N}(x)$ if $p>p_{*}$, and it is a local maximum for $p<p_{*}$. The value $p_{*}$ can be found as a solution of the equation

$$
4\left(p_{*}+1\right) \lambda\left(p_{*}+2 ; N\right)-\pi^{2} \lambda\left(p_{*} ; N\right)=0 .
$$

The lower and upper bounds for $p_{*}$ are:

$$
\begin{aligned}
p_{*} \geq & \frac{1}{8}\left(\pi^{2}(2 N+1)^{2}-12+\sqrt{\left(\pi^{2}(2 N+1)^{2}-12\right)^{2}-128}\right) \\
p_{*} \leq & \frac{1}{4} \sqrt{72 \pi^{2}(N+1)^{2}\left(2 \pi^{2}(N+1)^{2}-2-\pi^{2}\right)+4+36 \pi^{2}+9 \pi^{4}} \\
& +3 \pi^{2}(N+1)^{2}-\frac{3}{4}\left(2+\pi^{2}\right) .
\end{aligned}
$$

Proof. Let us consider

$$
\begin{aligned}
\mathfrak{h}_{p, N}^{\prime}(x)=p & \sin ^{p-1}(\pi x)(2 x-1)\left[\frac{\pi \cos (\pi x)}{2 x-1} \sum_{k=N+1}^{\infty}\left(\frac{1}{(k-x)^{p}}+\frac{1}{(k+x-1)^{p}}\right)\right. \\
& \left.+\frac{\sin (\pi x)}{2 x-1} \sum_{k=N+1}^{\infty}\left(\frac{1}{(k-x)^{p+1}}-\frac{1}{(k+x-1)^{p+1}}\right)\right]
\end{aligned}
$$


Here, the termwise differentiation of series (9) is legitimate because the series of derivatives $\mathfrak{h}_{p, N}^{\prime}(x)$ given by (13) converges uniformly.

Now, we will study (13) when $x \downarrow 1 / 2$. For all $x \in[1 / 2,1]$ we have that $p \sin ^{p-1}(\pi x)(2 x-1) \geq 0$. Letting $x \downarrow 1 / 2$ the following limits appear in (13):

$$
\begin{aligned}
\frac{\pi \cos (\pi x)}{2 x-1} \sum_{k=N+1}^{\infty}\left(\frac{1}{(k-x)^{p}}+\frac{1}{(k+x-1)^{p}}\right) & \rightarrow-\sum_{k=N+1}^{\infty} \frac{\pi^{2}}{(k-1 / 2)^{p}} ; \\
\frac{\sin (\pi x)}{2 x-1} \sum_{k=N+1}^{\infty}\left(\frac{1}{(k-x)^{p+1}}-\frac{1}{(k+x-1)^{p+1}}\right) & \rightarrow(p+1) \sum_{k=N+1}^{\infty} \frac{1}{(k-1 / 2)^{p+2}} .
\end{aligned}
$$

Obviously, $\mathfrak{h}_{p, N}^{\prime}(1 / 2)=0$. We are interested in the nature of the extremum at $x=1 / 2$. Hence, from (13), by L'Hôspital rule one concludes

$$
\mathfrak{h}_{p, N}^{\prime \prime}(1 / 2)=2 \lim _{x \downarrow 1 / 2} \frac{\mathfrak{h}_{p, N}^{\prime}(x)}{2 x-1}=\sum_{k=N+1}^{\infty} \frac{2 p(p+1)}{(k-1 / 2)^{p+2}}-\sum_{k=N+1}^{\infty} \frac{2 p \pi^{2}}{(k-1 / 2)^{p}} .
$$

To investigate (14) we take the following auxiliary result. Namely, let $\kappa \in$ $\mathbb{R}_{+} \backslash\{1\}$ and let $a: \mathbb{R}_{+} \rightarrow \mathbb{R}_{+}$be a positive monotone increasing function. Then

$$
\int_{a(1)}^{\infty} \frac{\left[a^{-1}(x)\right]}{x^{\kappa}} \mathrm{d} x=\frac{1}{\kappa-1} \sum_{n=1}^{\infty} \frac{1}{a^{\kappa-1}(n)},
$$

where $[\alpha]$ denotes the integer part of $\alpha$ and $a^{-1}(x)$ is the inverse function to $a(x),[13, \S 8]$. Now, let us rewrite (14) in the form

$$
\mathfrak{h}_{p, N}^{\prime \prime}(1 / 2)=2 p(p+1) \sum_{n=1}^{\infty} \frac{1}{(n+N-1 / 2)^{p+2}}-2 p \pi^{2} \sum_{n=1}^{\infty} \frac{1}{(n+N-1 / 2)^{p}} .
$$

By specifying $a(x) \equiv x+N-1 / 2, a^{-1}(x)=x-N+1 / 2$ we find from (15) that

$$
\mathfrak{h}_{p, N}^{\prime \prime}(1 / 2)=2 p^{2} \int_{N+1 / 2}^{\infty} \frac{[x-N+1 / 2]}{x^{p+1}}\left(\frac{(p+2)\left(1+p^{-1}\right)}{x^{2}}-\pi^{2}\right) \mathrm{d} x .
$$

Hence, if

$$
\frac{(p+2)\left(1+p^{-1}\right)}{(N+1 / 2)^{2}}-\pi^{2}<0,
$$

the inequality $\mathfrak{h}_{p, N}^{\prime \prime}(1 / 2)<0$ shows that the value $\mathfrak{h}_{p, N}(1 / 2)$ is a local maximum of $\mathfrak{h}_{p, N}(x)$. The inequality (17) holds for $p=1$, the expression $(p+2)\left(1+p^{-1}\right)$ decreases if $p \in[1, \sqrt{2})$ and increases if $p \in(\sqrt{2}, \infty)$. Solving (17) with respect to $p$ we get

$$
p<\frac{1}{8}\left(\pi^{2}(2 N+1)^{2}-12+\sqrt{\left(\pi^{2}(2 N+1)^{2}-12\right)^{2}-128}\right) .
$$

Thus, if $p_{*}$ exists, the lower bound on $p_{*}$ follows from the last inequality. 
Now, we are looking for values of $p, N$ which ensure a local minimum at $x=1 / 2$. Considering (16) once more, it is sufficient to find when

$$
\int_{N+1 / 2}^{\infty} \frac{[x-N+1 / 2]}{x^{3}}\left(\frac{(p+2)\left(1+p^{-1}\right)}{x^{2}}-\pi^{2}\right) \frac{\mathrm{d} x}{x^{p-2}}>0 .
$$

Fixing $N \in \mathbb{N}$ we remark that for some $p_{0}>\sqrt{2}$ there holds

$$
(p+2)\left(1+p^{-1}\right) \int_{N+1 / 2}^{\infty} \frac{[x-N+1 / 2]}{x^{5}} \mathrm{~d} x>\pi^{2} \int_{N+1 / 2}^{\infty} \frac{[x-N+1 / 2]}{x^{3}} \mathrm{~d} x,
$$

when $p>p_{0}$, since both integrals are positive and finite, and $(p+2)\left(1+p^{-1}\right)$ increases in $p$ when $p>\sqrt{2}$. Hence

$$
\int_{N+1 / 2}^{\infty} \frac{[x-N+1 / 2]}{x^{3}}\left(\frac{(p+2)\left(1+p^{-1}\right)}{x^{2}}-\pi^{2}\right) \mathrm{d} x>0 \quad\left(p>p_{0}\right) .
$$

Indeed, the integrand in (20) changes the sign from positive to negative only once on $\mathbb{R}_{+}$. Therefore, multiplying the integrand by the decreasing function $x^{2-p}$ does not change the sign of the integral (20) for $p>\max \left(p_{0}, 2\right)$. Thus (18) definitely holds for all $p \geq 2$ which satisfy (19).

Now, we have

$$
\frac{\int_{N+1 / 2}^{\infty} \frac{[x-N+1 / 2]}{x^{3}} \mathrm{~d} x}{\int_{N+1 / 2}^{\infty} \frac{[x-N+1 / 2]}{x^{5}} \mathrm{~d} x}<\frac{\int_{N+1 / 2}^{\infty} \frac{x-N+1 / 2}{x^{3}} \mathrm{~d} x}{\int_{N+1 / 2}^{\infty} \frac{x-N-1 / 2}{x^{5}} \mathrm{~d} x}=\frac{3}{2}\left(4(N+1)^{2}-1\right)=: A(N)
$$

and it follows easily that (18) holds for any $p$ satisfying the inequality

$$
B(p):=(p+2)\left(1+p^{-1}\right)>\frac{3 \pi^{2}\left(4(N+1)^{2}-1\right)}{2} .
$$

Indeed, bearing in mind the equivalent form of (21), that is

$$
\int_{N+1 / 2}^{\infty} \frac{[x-N+1 / 2]}{x^{5}} \mathrm{~d} x>\frac{1}{A(N)} \int_{N+1 / 2}^{\infty} \frac{[x-N+1 / 2]}{x^{3}} \mathrm{~d} x,
$$

and re-writing (20) in the form

$$
\begin{aligned}
B(p) \int_{N+1 / 2}^{\infty} \frac{[x-N+1 / 2]}{x^{5}} \mathrm{~d} x-\pi^{2} \int_{N+1 / 2}^{\infty} \frac{[x-N+1 / 2]}{x^{3}} \mathrm{~d} x \\
>\left(\frac{B(p)}{A(N)}-\pi^{2}\right) \int_{N+1 / 2}^{\infty} \frac{[x-N+1 / 2]}{x^{3}} \mathrm{~d} x>0,
\end{aligned}
$$

we deduce the condition (22). (Moreover, $p>2$ follows from this inequality). Thus, solving the inequality (22) with respect to $p$ we conclude that, since $\mathfrak{h}_{p, N}^{\prime \prime}(1 / 2)>0$ for

$$
\begin{gathered}
p \geq \frac{1}{4} \sqrt{72 \pi^{2}(N+1)^{2}\left(2 \pi^{2}(N+1)^{2}-2-\pi^{2}\right)+4+36 \pi^{2}+9 \pi^{4}} \\
+3 \pi^{2}\left((N+1)^{2}-\frac{1}{4}\right)-\frac{3}{2},
\end{gathered}
$$

the function $\mathfrak{h}_{p, N}(x)$ possesses a local minimum at $x=1 / 2$. So, if $p_{*}$ exists, the upper bound on $p_{*}$ clearly follows from the last inequality. 
Now, we prove the existence of $p_{*}$. From the previous considerations it follows that for some values of $p$ there is a local maximum in $x=1 / 2$, for others there is a local minimum. Therefore, due to the continuity at $p$ of the left part of (18), we find that there exists at least one $p_{*}$ for which

$$
\int_{N+1 / 2}^{\infty} \frac{[x-N+1 / 2]}{x^{p_{*}+1}}\left(\frac{\left(p_{*}+2\right)\left(1+p_{*}^{-1}\right)}{x^{2}}-\pi^{2}\right) \mathrm{d} x=0 .
$$

By (17) we have that $p_{*}>\sqrt{2}$. On the other hand as $(p+2)\left(1+p^{-1}\right)$ increases, for $p>p_{*}$ we have

$$
\int_{N+1 / 2}^{\infty} \frac{[x-N+1 / 2]}{x^{p_{*}+1}}\left(\frac{(p+2)\left(1+p^{-1}\right)}{x^{2}}-\pi^{2}\right) \mathrm{d} x \geq 0 .
$$

As the integrand changes the sign from positive to negative only once on $\mathbb{R}_{+}$ multiplying the integrand by the decreasing function $x^{p_{*}-p}$ we get

$$
\int_{N+1 / 2}^{\infty} \frac{[x-N+1 / 2]}{x^{p+1}}\left(\frac{(p+2)\left(1+p^{-1}\right)}{x^{2}}-\pi^{2}\right) \mathrm{d} x>0 .
$$

Therefore, if $x=1 / 2$ is the abscissa of a local minimum for some $p_{1}$ then for all $p>p_{1}$ there is a local minimum at $x=1 / 2$ as well. This shows the uniqueness of $p_{*}$ which is a root of (14). The final step in the proof is to notice that the right-hand expression in (14) can be represented as

$$
\sum_{k=N+1}^{\infty} \frac{2 p(p+1)}{(k-1 / 2)^{p+2}}-\sum_{k=N+1}^{\infty} \frac{2 p \pi^{2}}{(k-1 / 2)^{p}}=2^{p+1} p\left(4(p+1) \lambda(p+2 ; N)-\pi^{2} \lambda(p ; N)\right) .
$$

This finishes the proof of Theorem 3.

Corollary 3.1. $\quad$ a) $\left(1 / 2, \mathfrak{h}_{p, N}(1 / 2)\right)$ is a local maximum by (17) if

$$
N \geq \frac{1}{\pi} \sqrt{(p+2)\left(1+p^{-1}\right)}-\frac{1}{2} \text {. }
$$

b) By the previous estimate $\left(1 / 2, \mathfrak{h}_{p, N}(1 / 2)\right)$ is a local maximum for arbitrary $N \in \mathbb{N}$ if $\sqrt{(p+2)\left(1+p^{-1}\right)} \leq 3 \pi / 2$, i.e.

$$
p \leq \frac{1}{8}\left(9 \pi^{2}+\sqrt{16-216 \pi^{2}+81 \pi^{4}}-12\right) \approx 19.1019 .
$$

Remark 2. The case b) in Corollary 3.1 covers the particular case $p=2$ discussed in [12].

We need some bounds for $p_{*}$ in solving numerically the transcendental equation (10).

Remark 3. For certain $N$ the corresponding values of $p_{*}$ and their bounds given in Theorem 3 , are shown in the table: 


\begin{tabular}{|c|c|c|c|c|}
\hline$N$ & 1 & 2 & 3 & 4 \\
\hline$p_{*}$ & 21.2069 & 60.685 & 119.903 & 198.859 \\
lower bound (11) & 19.1019 & 58.6509 & 117.8857 & 196.8493 \\
upper bound (12) & 219.057 & 515.1504 & 929.6755 & 1462.6349 \\
\hline
\end{tabular}

One can see that the lower bounds correspond to exact values of $p_{*}$. However, the upper bounds are rough. But they can be sharpened by, for example, following the previous method of calculation. One considers

$$
(p+2)\left(1+p^{-1}\right) \int_{N+1 / 2}^{\infty} \frac{[x-N+1 / 2]}{x^{4+\varepsilon}} \mathrm{d} x>\pi^{2} \int_{N+1 / 2}^{\infty} \frac{[x-N+1 / 2]}{x^{2+\varepsilon}} \mathrm{d} x
$$

instead of (19) where $p-1>\varepsilon>0$, then minimizes the value of the upper bound for $p_{*}$ with respect to some admissible $\varepsilon$.

Remark 4. Due to numerical results in Remark 3 the estimate in Corollary 3.1 b) can be improved to $p \leq 21.2069$.

Now, we are interested in properties of (9) at an arbitrary point $x$. Let us denote the $k$-th term of (9) by $\psi_{k}(x), k \geq 2$, i.e.

$$
\mathfrak{h}_{p, N}(x)=\sin ^{p}(\pi x) \sum_{k=N+1}^{\infty}\left(\frac{1}{(k-x)^{p}}+\frac{1}{(k+x-1)^{p}}\right)=: \sum_{k=N+1}^{\infty} \psi_{k}(x) .
$$

Lemma 2. For $x \in(1 / 2,1)$, from $\psi_{k}^{\prime}(x)<0$ it follows that $\psi_{k+1}^{\prime}(x)<0$.

Proof. Using the well-known identity

$$
\frac{\Gamma(s)}{\alpha^{s}}=\int_{0}^{\infty} e^{-\alpha t} t^{s-1} \mathrm{~d} t
$$

specifying $\alpha=k-x$, then $k+x-1$ we get

$$
\begin{array}{r}
\psi_{k}^{\prime}(x)=p \sin ^{p-1}(\pi x)\left[\pi \cos (\pi x)\left(\frac{1}{(k-x)^{p}}+\frac{1}{(k+x-1)^{p}}\right)\right. \\
\left.+\sin (\pi x)\left(\frac{1}{(k-x)^{p+1}}-\frac{1}{(k+x-1)^{p+1}}\right)\right] \\
=\frac{p \sin ^{p-1}(\pi x)}{\Gamma(p)} \int_{0}^{\infty} e^{-k t} t^{p-1}\left(\pi \cos (\pi x)\left(e^{x t}+e^{(1-x) t}\right)\right. \\
\left.+\frac{t \sin (\pi x)}{p}\left(e^{x t}-e^{(1-x) t}\right)\right) d t=: \int_{0}^{\infty} g_{k}(t) \mathrm{d} t
\end{array}
$$

Therefore

Denote

$$
\psi_{k+1}^{\prime}(x)=\int_{0}^{\infty} e^{-t} g_{k}(t) \mathrm{d} t
$$

$$
\widetilde{g}_{k}(t):=\pi \cos (\pi x)\left(e^{x t}+e^{(1-x) t}\right)+\frac{t \sin (\pi x)}{p}\left(e^{x t}-e^{(1-x) t}\right) .
$$


Let us show that there exists $t_{0}>0$ such that $\widetilde{g}_{k}(t) \leq 0,0 \leq t \leq t_{0}$, and $\widetilde{g}_{k}(t) \geq$ $0, t \geq t_{0}$. To do this, let us note that $\widetilde{g}_{k}(0)=2 \pi \cos (\pi x)<0, x \in(1 / 2,1)$. Then rewrite $\widetilde{g}_{k}(t)$ in the form

$$
\widetilde{g}_{k}(t)=p^{-1} t \sin (\pi x)\left(e^{x t}+e^{(1-x) t}\right)\left(\pi p t^{-1} \cot (\pi x)+\frac{2}{1+e^{(1-2 x) t}}-1\right) .
$$

The term $p^{-1} t \sin (\pi x)\left(e^{x t}+e^{(1-x) t}\right)$ is positive for all $x \in(1 / 2,1), t>0$. For fixed $x \in(1 / 2,1)$ the function $\pi p t^{-1} \cot (\pi x)$ increases from $-\infty$ to 0 and the function $\frac{2}{1+e^{(1-2 x) t}}-1$ increases from 0 to 1 when $t$ runs over the positive halfaxis. Hence, there exists a unique $t_{0}$ such that $\widetilde{g}_{k}\left(t_{0}\right)=0$.

By the relation

$$
g_{k}(t)=\frac{p \sin ^{p-1}(\pi x)}{\Gamma(p)} e^{-k t} t^{p-1} \widetilde{g}_{k}(t)
$$

we deduce that $g_{k}(t) \leq 0,0 \leq t \leq t_{0}$, and $g_{k}(t) \geq 0, t \geq t_{0}$. Therefore, since $\psi_{k}^{\prime}(x)=\int_{0}^{\infty} g_{k}(t) \mathrm{d} t<0$ we conclude that $\psi_{k+1}^{\prime}(x)=\int_{0}^{\infty} e^{-t} g_{k}(t) \mathrm{d} t<0$ since $e^{-t}$ is a decreasing function.

For further investigations we need the next two lemmata.

Lemma 3. For each $z \in[0,1 / 2]$ we have

$$
\frac{\sin (2 \pi z)}{2 \pi z} \leq \frac{1-z^{2}}{1+z^{2}}
$$

Proof. From the series expansion for the sine function we have

$$
\frac{\sin (2 \pi z)}{2 \pi z} \leq 1-\frac{(2 \pi z)^{2}}{3 !}+\frac{(2 \pi z)^{4}}{5 !}
$$

as a consequence of $\frac{(2 \pi z)^{2 k}}{(2 k+1) !} \geq \frac{(2 \pi z)^{2 k+2}}{(2 k+3) !}, k \in \mathbb{N}$, which holds for all $z \in[0,1 / 2]$. Thus, to prove (24), it is enough to show that

$$
1-\frac{(2 \pi z)^{2}}{3 !}+\frac{(2 \pi z)^{4}}{5 !} \leq \frac{1-z^{2}}{1+z^{2}}
$$

On putting $z^{2}=t$, (25) can be rewritten as

$$
\frac{t}{1+t} \leq \frac{\pi^{2} t}{3}-\frac{\pi^{4} t^{2}}{15}
$$

or

$$
5 \pi^{2}-15+\left(5 \pi^{2}-\pi^{4}\right) t-\pi^{4} t^{2} \geq 0 .
$$

For $t \in[0,1 / 4]$ the last inequality holds true, because the polynomial $5 \pi^{2}-$ $15+\left(5 \pi^{2}-\pi^{4}\right) t-\pi^{4} t^{2}$ has two real roots at $t_{1} \approx-0.9$, and $t_{2} \approx 0.39$.

Lemma 4. The abscissa of extrema of $\psi_{k}(x)$ are $1 / 2$ and 1 for all admissible p. Moreover, if $p>\pi^{2}(k-1 / 2)^{2}-1, \psi_{k}(x)$ attains an extremum at some $x_{k}^{*} \in\left(1 / 2, A_{k}\right), A_{k} \in(1 / 2,1)$ being the unique solution of the equation

$$
\cot (\pi x)=-\frac{1}{\pi(k-x)}
$$


Each $x_{k}^{*} \in\left(1 / 2, A_{k}\right)$ is the abscissa of an extremum corresponding to a unique $\widetilde{p}$ which satisfies

$$
=\frac{\ln \frac{1-\pi \cot \left(\pi x_{k}^{*}\right)\left(k+x_{k}^{*}-1\right)}{\pi \cot \left(\pi x_{k}^{*}\right)\left(k+x_{k}^{*}-1\right)-1+\frac{2 k-1}{k-x_{k}^{*}}}}{\ln \left(k+x_{k}^{*}-1\right)-\ln \left(k-x_{k}^{*}\right)}>1 .
$$

Proof. We immediately obtain by (23) that $\psi_{k}^{\prime}(x)$ vanishes at $1 / 2$ and 1 . Another possible extremum could arise from solutions of the equation

$$
\pi \cot (\pi x)\left(\frac{1}{(k-x)^{p}}+\frac{1}{(k+x-1)^{p}}\right)=\frac{1}{(k+x-1)^{p+1}}-\frac{1}{(k-x)^{p+1}}
$$

where $x \in(1 / 2,1)$. Let us show that (26) has one only solution. Simple transformations of (26) give

$$
\frac{(k+x-1)^{p+1}-(k-x)^{p+1}}{\left((k+x-1)^{p}+(k-x)^{p}\right)(x-1 / 2)}=\frac{-\pi \cot (\pi x)(k+x-1)(k-x)}{(x-1 / 2)} .
$$

On putting $z=x-1 / 2 \in(0,1 / 2)$, and $a=k-1 / 2>0$, (27) becomes

$$
L(z):=\frac{(a+z)^{p+1}-(a-z)^{p+1}}{z\left((a+z)^{p}+(a-z)^{p}\right)}=\frac{\pi \tan (z \pi)\left(a^{2}-z^{2}\right)}{z}=: R(z) .
$$

We now differentiate the left-hand member of (28) to obtain

$$
L^{\prime}(z)=-\frac{\left(\frac{a+z}{a-z}\right)^{2 p}-1-p\left(\left(\frac{a+z}{a-z}\right)^{2}-1\right)\left(\frac{a+z}{a-z}\right)^{p-1}}{z^{2}\left(\left(\frac{a+z}{a-z}\right)^{p}+1\right)^{2}} .
$$

Now, it is not hard to show that for each $t \geq 1, p \geq 1$ we have

$$
t^{2 p}-1 \geq p\left(t^{2}-1\right) t^{p-1} .
$$

On taking $t=\frac{a+z}{a-z}$ in (29), inequality 30 shows that $L(z)$ decreases in $z$.

We now differentiate the right-hand member of (28) to obtain

$$
\begin{aligned}
R^{\prime}(z) & =\frac{\pi\left(a^{2}+z^{2}\right)}{z \cos ^{2}(\pi z)}\left(1-\frac{2 z^{2}}{a^{2}+z^{2}}-\frac{\sin (2 \pi z)}{2 \pi z}\right) \\
& \geq \frac{\pi\left(a^{2}+z^{2}\right)}{z \cos ^{2}(\pi z)}\left(1-\frac{2 z^{2}}{1+z^{2}}-\frac{\sin (2 \pi z)}{2 \pi z}\right) .
\end{aligned}
$$

It follows from (31) and Lemma 3 that $R(z)$ increases in $z$.

Letting $z \rightarrow 1 / 2$ we get

$$
\lim _{z \rightarrow 1 / 2} L(z)=2 \frac{(a+1 / 2)^{p+1}-(a-1 / 2)^{p+1}}{(a+1 / 2)^{p}+(a-1 / 2)^{p}}, \quad \lim _{z \rightarrow 1 / 2} R(z)=\infty ;
$$

and similarly

$$
\lim _{z \rightarrow 0} L(z)=p+1, \quad \lim _{z \rightarrow 0} R(z)=(a \pi)^{2} .
$$


By the aforementioned properties of $L(z)$ and $R(z)$ we find that there are only two possibilities: (i) (28) has no solutions, or (ii) it has a unique solution on the interval $(0,1 / 2)$. Moreover, (28) has a solution on the interval $z \in(0,1 / 2)$ if and only if $p+1>\pi^{2} a^{2}=\pi^{2}(k-1 / 2)^{2}$.

Further simple transformations of (26) give

$$
\left(1+\frac{2 x-1}{k-x}\right)^{p}=\frac{1-\pi \cot (\pi x)(k+x-1)}{\pi \cot (\pi x)(k+x-1)-1+\frac{2 k-1}{k-x}} .
$$

The expression on the left side of (32) increases unboundedly with growing $p$. Therefore, $x_{k}^{*} \in(1 / 2,1)$ might be an extremal point for one $p$ only. From (32) we easily calculate this value

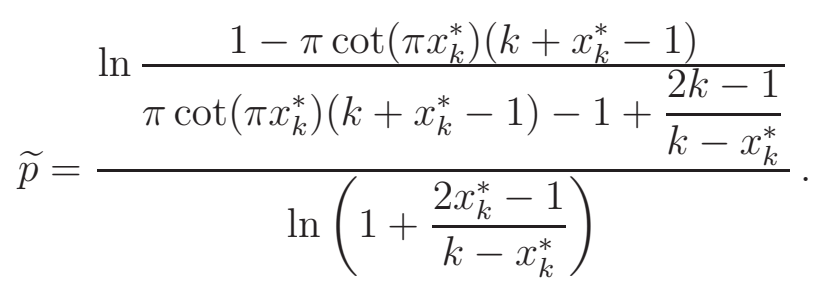

Let us show that $x_{k}^{*}$ cannot be greater than $A_{k}$. The denominator in (33) is defined on the whole of $(1 / 2,1)$. To investigate the numerator we study the equation

$$
\pi \cot (\pi x)(k+x-1)-1+\frac{2 k-1}{k-x}=0
$$

that is

$$
\cot (\pi x)=-\frac{1}{\pi(k-x)}
$$

Because of

$$
0=\cot (\pi / 2)>-\frac{1}{\pi(k-1 / 2)}, \quad-\infty=\lim _{x \rightarrow 1} \cot (\pi x)<-\frac{1}{\pi(k-1)},
$$

and

$$
\cot ^{\prime}(\pi x)<\left(-\frac{1}{\pi(k-x)}\right)^{\prime} \quad(x \in(1 / 2,1))
$$

it is obvious that the equation (34) has an unique solution in $(1 / 2,1)$ which we denote by $A_{k}$. Of course, $x_{k}^{*} \in\left(1 / 2, A_{k}\right)$, because $\pi \cot (\pi x)(k+x-1)-1+\frac{2 k-1}{k-x}<$ 0 for $x>A_{k}$.

Let us show that for arbitrary $x \in\left(1 / 2, A_{k}\right)$ we have

$$
\ln \left(-1+\frac{\frac{2 k-1}{k-x}}{\pi \cot (\pi x)(k+x-1)-1+\frac{2 k-1}{k-x}}\right)>0
$$

and the value $\widetilde{p}$, given above by (33), is greater than 1 .

Because of (33), (35) becomes equivalent to

$$
-1+\frac{\frac{2 k-1}{k-x}}{\pi \cot (\pi x)(k+x-1)-1+\frac{2 k-1}{k-x}}>1+\frac{2 x-1}{k-x},
$$


that is, to

$$
2-\frac{2 k-1}{k-x}>\pi \cot (\pi x)(k+x-1) .
$$

For $x=1 / 2$, (36) becomes an identity. Note that for $x \in\left(1 / 2, A_{k}\right)$ the derivatives of both sides of (36) satisfy the inequality

$$
\left(2-\frac{2 k-1}{k-x}\right)^{\prime}=-\frac{2 k-1}{(k-x)^{2}}>\pi\left(\cot (\pi x)-\frac{\pi(k+x-1)}{\sin ^{2}(\pi x)}\right)=\pi\left(\frac{k+x-1}{\tan (\pi x)}\right)^{\prime}
$$

which is satisfied since

$$
\sin ^{2}(\pi x)<\frac{\pi^{2}}{2} \cdot \frac{k+x-1}{k-1 / 2} \cdot(k-x)^{2} \quad(x \in(1 / 2,1), k \geq 2) .
$$

Therefore, $\widetilde{p}$ is correctly defined.

Corollary 3.2. For $p \leq \pi^{2}(k-1 / 2)^{2}-1$ the function $\psi_{k}(x)$ decreases on $[1 / 2,1]$.

Proof. The statement is a consequence of Lemma 4 and the fact $\psi_{k}(1 / 2)>$ $\psi_{k}(0)=0$.

Theorem 4. For $p \leq \pi^{2}(N+1 / 2)^{2}-1$ we have

$$
\sum_{n \in \mathbb{Z} \backslash \mathfrak{J} x}|\operatorname{sinc}(x-n)|^{p} \leq 2\left(\frac{2}{\pi}\right)^{p} \lambda(p ; N) .
$$

The inequality is sharp and becomes an equality at $x=1 / 2$.

Proof. The assertion is an easy consequence of Lemma 2, Corollary 3.2 and the identity

$$
\sum_{n \in \mathbb{Z} \backslash \mathfrak{J}_{1 / 2}}|\operatorname{sinc}(1 / 2-n)|^{p}=2\left(\frac{2}{\pi}\right)^{p} \sum_{k=N+1}^{\infty} \frac{1}{(2 k-1)^{p}}=2\left(\frac{2}{\pi}\right)^{p} \lambda(p ; N) .
$$

Remark 5. The result of Theorem 4 improves the lower bound (11) in Theorem 3. For some positive integer $N$ the numerical values of $\pi^{2}(N+1 / 2)^{2}-1$ are shown in the table (compare with the table in Remark 3):

\begin{tabular}{|c|c|c|c|c|}
\hline$N$ & 1 & 2 & 3 & 4 \\
\hline$\pi^{2}(N+1 / 2)^{2}-1$ & 21.2066 & 60.6849 & 119.902 & 198.859 \\
\hline
\end{tabular}

Remark 6. The expression $\pi^{2}(N+1 / 2)^{2}-1$ grows quickly in $N$ and at the same time covers a wide range of small $p$ values. Therefore, the estimate (37) is important for applications. 


\section{Upper bounds without known signal decay rate}

Here, and in what follows let $(p, q)$ be a positive Hölder pair, i.e. $p^{-1}+q^{-1}=1$, $p>1$.

The most frequently appearing rearrangement of (2) in the literature is of the form

$$
\left\|T_{\mathfrak{J}}(f ; \mathbf{x})\right\|_{\infty} \leq\left(\sum_{\mathbf{n} \in \mathbb{Z}^{d} \backslash \mathfrak{J}}\left|S\left(\mathbf{x}, t_{\mathbf{n}}\right)\right|^{p}\right)^{1 / p}\left(\sum_{\mathbf{n} \in \mathbb{Z}^{d} \backslash \mathfrak{J}}\left|f\left(t_{\mathbf{n}}\right)\right|^{q}\right)^{1 / q}=: A_{p} B_{q} .
$$

To make the approximant $Y_{\mathfrak{J}}(f ; \mathbf{x})$ more precise it is of interest to assume $\mathfrak{J}=$ $\mathfrak{J}_{\mathbf{x}}$, i.e. that the index set of sampling restoration $\mathfrak{J}_{\mathbf{x}}$ depends on the location of $\mathbf{x}$ with respect to the behaviour of $f$ when estimating $B_{q}$. That means $T_{\mathfrak{J}_{\mathbf{x}}}(f ; \mathbf{x})$ is a fortiori time shifted and possesses time adapted sampling size. Thus our approach is closer to an interpolation rather than to extrapolation procedure. The earlier works [14, 12, 15] did not mention these facts.

Using the classical approach one operates with the straightforward estimate $B_{q} \leq C_{f, \mathfrak{J}}\|f\|_{q}$ where $C_{f, \mathfrak{J}}$ is a suitable absolute constant. Therefore (38) becomes

$$
\left\|T_{\mathfrak{J}_{\mathbf{x}}}(f ; \mathbf{x})\right\|_{\infty} \leq A_{p} C_{f, \mathfrak{J}_{\mathbf{x}}}\|f\|_{q}
$$

where $C_{f, \mathfrak{J}_{\mathbf{x}}}$ depends on the properties of the signal function and vanishes when $\left|\mathfrak{J}_{\mathbf{x}}\right| \rightarrow \infty$.

To obtain a class of truncation error upper bounds when the decay rate of the initial signal function is not known we are interested in estimates for $A_{p}$ which vanish with $\left|\mathfrak{J}_{\mathbf{x}}\right| \rightarrow \infty$, and estimates like $C_{f, \mathfrak{J}_{\mathbf{x}}} \leq C_{f}$ which are valid for all $\mathbf{x}$.

We will consider the case $\mathfrak{T}=\mathbb{Z}^{d}$. Let us specify the sampling function

$$
S\left(\mathbf{x}, t_{\mathbf{n}}\right)=\prod_{j=1}^{d} \operatorname{sinc}\left(x_{j}-n_{j}\right)
$$

where $\mathbf{n}=\left(n_{1}, \ldots, n_{d}\right) \in \mathbb{Z}^{d}$. Then (1) becomes the equally sampled (regular) uniformly convergent WKS sampling formula

$$
f(\mathbf{x})=\sum_{\mathbf{n} \in \mathbb{Z}^{d}} f(\mathbf{n}) \prod_{j=1}^{d} \operatorname{sinc}\left(x_{j}-n_{j}\right) \quad\left(\mathbf{x} \in \mathbb{R}^{d}\right) .
$$

Let $\mathbf{N}:=\left(N_{1}, \cdots, N_{d}\right) \in \mathbb{N}^{d}, \mathfrak{J}_{\mathbf{x}}:=\left\{\mathbf{n}: \bigwedge_{j=1}^{d}\left(\left|x_{j}-n_{j}\right| \leq N_{j}\right)\right\}$. Then the WKS sum truncated to $\mathfrak{J}_{\mathbf{x}}$ reads

$$
Y_{\mathfrak{J}_{\mathbf{x}}}(f ; \mathbf{x})=\sum_{\mathbf{n} \in \mathfrak{J}_{\mathbf{x}}} f(\mathbf{n}) \prod_{j=1}^{d} \operatorname{sinc}\left(x_{j}-n_{j}\right)
$$

and one introduces the consequent truncation error by

$$
\left\|T_{\mathbf{N}, d}(f ; \mathbf{x})\right\|_{\infty}:=\left\|f(\mathbf{x})-Y_{\mathfrak{J}_{\mathbf{x}}}(f ; \mathbf{x})\right\|_{\infty} .
$$

In the sequel let us define $\widetilde{N}:=\min _{j=1, \cdots, d} N_{j}$ and $\boldsymbol{\pi}=(\pi, \ldots, \pi)_{1 \times d}$. 
Theorem 5. Let $f \in B_{\pi, d}^{q}, q \geq 1+\left(\pi^{2}(\tilde{N}+1 / 2)^{2}-2\right)^{-1}$. Then we have

$$
\left\|T_{\mathbf{N}, d}(f ; \mathbf{x})\right\|_{\infty} \leq C(\mathbf{N}, d, q) \cdot\|f\|_{q},
$$

where

$$
C(\mathbf{N}, d, q):=\frac{2^{2-\frac{1}{q}}}{\pi} \mathfrak{C}_{\frac{q}{q-1}}^{(d-1)\left(1-\frac{1}{q}\right)} \mathfrak{B}_{d, q}^{1 / q}\left(\sum_{j=1}^{d} \lambda\left(\frac{q}{q-1} ; N_{j}\right)\right)^{1-\frac{1}{q}} ;
$$

and the constants $\mathfrak{C}_{\alpha}, \mathfrak{B}_{d, \beta}$ are introduced in Theorem 2 and by (15) respectively.

Proof. Now $f \in B_{\pi, d}^{q}$, hence, by Lemma 1, $f\left(x_{1}, \ldots, x_{k-1}, \cdot, x_{k+1}, \ldots, x_{d}\right) \in B_{\pi, 1}^{q}$ for all $k=1, \cdots, d$. So, the direct $d$-dimensional generalization of the proof given for the two-dimensional case in [3, §48] shows that (39) is valid, that is

$$
\left\|T_{\mathbf{N}, d}(f ; \mathbf{x})\right\|_{\infty}=\left\|\sum_{\mathbf{n} \in \mathbb{Z}^{d} \backslash \mathfrak{J}_{\mathbf{x}}} f(\mathbf{n}) \prod_{j=1}^{d} \operatorname{sinc}\left(x_{j}-n_{j}\right)\right\|_{\infty} .
$$

Hence, it is not hard to see that for a Hölder pair $(p, q)$ we have

$$
\left\|T_{\mathbf{N}, d}(f ; \mathbf{x})\right\|_{\infty} \leq\left(\sum_{\mathbf{n} \in \mathbb{Z}^{d} \backslash \mathfrak{J}_{\mathbf{x}}} \prod_{j=1}^{d}\left|\operatorname{sinc}\left(x_{j}-n_{j}\right)\right|^{p}\right)^{1 / p}\left(\sum_{\mathbf{n} \in \mathbb{Z}^{d} \backslash \mathfrak{J}_{\mathbf{x}}}|f(\mathbf{n})|^{q}\right)^{1 / q} .
$$

By Theorem 1 the multidimensional Plancherel-Pólya inequality (4) holds for functions $f$ belonging to the Bernstein space $B_{\pi, d}^{q}, q \geq 1$ and since in the regular sampling case one has $\delta_{\ell}=1, \ell=1, \cdots, d$, that is $\mathfrak{T} \equiv \mathbb{Z}^{d}$. It follows that the second term in (41) is bounded by $\mathfrak{B}_{d, q}^{1 / q}\|f\|_{q}$. Indeed, we easily deduce

$$
\sum_{\mathbf{n} \in \mathbb{Z}^{d} \backslash \mathfrak{J}_{\mathbf{x}}}|f(\mathbf{n})|^{q} \leq \sum_{\mathbf{n} \in \mathbb{Z}^{d}}|f(\mathbf{n})|^{q} \leq \mathfrak{B}_{d, q}\|f\|_{q}^{q} .
$$

Now, we use the estimate

$$
\begin{aligned}
\sum_{\mathbf{n} \in \mathbb{Z}^{d} \backslash \mathfrak{J}_{\mathbf{x}}} & \prod_{j=1}^{d}\left|\operatorname{sinc}\left(x_{j}-n_{j}\right)\right|^{p} \leq \sum_{j=1}^{d} \sum_{n_{j} \in \mathbb{Z} \backslash \mathfrak{J}_{j}}\left|\operatorname{sinc}\left(x_{j}-n_{j}\right)\right|^{p} \prod_{k \neq j} \sum_{n_{k} \in \mathbb{Z}}\left|\operatorname{sinc}\left(x_{k}-n_{k}\right)\right|^{p} \\
& \leq\left(\sup _{x \in[1 / 2,1]} \sum_{n \in \mathbb{Z}}|\operatorname{sinc}(x-n)|^{p}\right)^{d-1} \sum_{j=1}^{d} \sum_{n_{j} \in \mathbb{Z} \backslash \mathfrak{J}_{x_{j}}}\left|\operatorname{sinc}\left(x_{j}-n_{j}\right)\right|^{p} .
\end{aligned}
$$

The application of (37) to the last term in (42) requires $p \leq \pi^{2}\left(N_{j}+1 / 2\right)^{2}-$ 1 for all $j=1, \cdots, d$. Rewriting all these constraints we obtain $q \geq 1+$ $\left(\pi^{2}(\widetilde{N}+1 / 2)^{2}-2\right)^{-1}$. So, as $\sigma_{\ell}=\pi, \delta_{\ell}=1$ we conclude

$\sum_{\mathbf{n} \in \mathbb{Z}^{d} \backslash \mathfrak{J} \mathbf{x}} \prod_{j=1}^{d}\left|\operatorname{sinc}\left(x_{j}-n_{j}\right)\right|^{p} \leq 2\left(\frac{2}{\pi}\right)^{p} \sum_{j=1}^{d} \lambda\left(p ; N_{j}\right)\left(\sup _{x \in[1 / 2,1]} \sum_{n \in \mathbb{Z}}|\operatorname{sinc}(x-n)|^{p}\right)^{d-1}$.

By applying Theorem 2 to this estimate, then substituting the values of all constants involved and noticing that $p^{-1}+q^{-1}=1,(p, q)$ being a positive Hölder pair, we complete the proof of the Theorem. 
Remark 7. For the particular case of equal truncation sizes $N_{1}=\ldots=N_{d}=$ $\widetilde{N}$ and $q_{*}:=1+\frac{1}{\pi^{2}(\widetilde{N}+1 / 2)^{2}-2}$ the corresponding numerical values of $C_{\mathbf{N}}:=$ $C(\mathbf{N}, d, q)$ are given in the table:

\begin{tabular}{|r|c|c|c|c|c|c|c|c|c|}
\hline$\widetilde{N}$ & \multicolumn{3}{|c|}{1} & \multicolumn{3}{c|}{2} & \multicolumn{3}{c|}{3} \\
\hline$q_{*}$ & \multicolumn{3}{|c|}{1.0495} & \multicolumn{3}{c|}{1.0168} & \multicolumn{3}{c|}{1.0084} \\
\hline$d$ & 1 & 2 & 3 & 1 & 2 & 3 & 1 & 2 & 3 \\
\hline$C_{\mathbf{N}}$ & 0.6727 & 2.1328 & 6.6703 & 0.3968 & 1.2369 & 3.8368 & 0.2822 & 0.8756 & 2.7103 \\
\hline
\end{tabular}

Remark 8. In the case $p=q=2$ the $L^{2}\left(\mathbb{R}^{d}\right)$-theory shows that the universal truncation error upper bound takes the form

$$
\begin{aligned}
\left\|T_{\mathbf{N}, d}(f, \mathbf{x})\right\|_{\infty} & \leq \sqrt{1-\prod_{j=1}^{d}\left(1-\frac{8}{\pi^{2}} \lambda\left(2 ; N_{j}\right)\right)} \cdot\|f\|_{2} \\
& =\sqrt{1-\frac{8^{d}}{\pi^{2 d}} \prod_{j=1}^{d} \sum_{n_{j}=1}^{N_{j}} \frac{1}{\left(2 n_{j}-1\right)^{2}}} \cdot\|f\|_{2} .
\end{aligned}
$$

This result is valid for all $\mathbf{N} \in \mathbb{N}^{d}$ and it is mainly simpler than the related restriction of (40). Moreover, this bound is sharp, see [1, Theorem 1].

Corollary 5.1. Let $f \in B_{\pi, d}^{q}, q>1$. The convergence rate in the multidimensional WKS sampling restoration procedure is of magnitude

$$
\left\|T_{\mathbf{N}, d}(f ; \mathbf{x})\right\|_{\infty}=\mathcal{O}\left(\widetilde{N}^{-1 / q}\right) \quad(\widetilde{N} \rightarrow \infty) .
$$

Proof. By the estimate

$$
\lambda(p ; N) \leq \frac{1}{(2 N+1)^{p}}+\int_{N+1}^{\infty} \frac{\mathrm{d} x}{(2 x-1)^{p}}=\frac{1}{(2 N+1)^{p-1}}\left(\frac{1}{2 N+1}+\frac{1}{2(p-1)}\right)
$$

we obtain $\lambda(p ; N)=\mathcal{O}\left(N^{-p+1}\right)$. Letting $\widetilde{N} \rightarrow \infty$ under the constraint $q \geq$ $1+\left(\pi^{2}(\widetilde{N}+1 / 2)^{2}-2\right)^{-1}$ we arrive at (43) with the aid of Theorem 5 ,

\section{Final remarks}

A. In the majority of articles devoted to Whittaker-Kotel'nikov-Shannon sampling theorems, their numerous generalizations, the related truncation error analysis and the sampling approximation convergence questions in the various $L^{p}\left(\mathbb{R}^{d}\right)$-type spaces (e.g. [10], [1], [16, and the references therein), the authors consider particular classes of functions with prescribed decay rates. These assumption upon the initial signal give one an opportunity to estimate the constant $B_{q}$ in (38) so, that the estimates vanish when the finite sampling restoration sum size parameter $N$ runs to infinity. The constant $A_{p}$ is another feature of interest here. It too was introduced in (38), and its novelty lies in the fact that one usually estimates by an absolute constant which does not depend on $N$. 
B. In this article we propose estimates for $A_{p}$ which depend on $N$ and tends to zero for $N$ growing. This approach enables us to consider and to obtain approximation error estimates for wide functional classes without strong assumptions upon the decay rate of initial signals. It seems that proposed estimates are the first attempts in sampling theory to analyze the properties of the series

$$
\mathfrak{h}_{p, N}(x):=\sum_{n \notin \mathfrak{J} x}|\operatorname{sinc}(x-n)|^{p}
$$

for $p \neq 2$ as functions of $x$. The proposed procedure treats the truncation error $T_{\mathfrak{J}_{\mathbf{x}}}(f ; \mathbf{x})$ in one and higher dimensions and it results in sharper estimates in comparison with known ones in the literature. Very interesting relations have been found on the dependence between the extremums of $\mathfrak{h}_{p, N}(x)$, its various properties and the joint behaviour of $N$ and $p$. New related truncation error upper bounds are derived in $\|\cdot\|_{\infty}$-norm for the sampling restoration procedure. Several numerical examples illustrates the matter in the multidimensional case too.

C. Moreover, the results obtained and the numerical simulations suggest some new hypotheses and open problems:

(1) To investigate the case of $p_{*}$ in Theorem 3 ,

(2) It seems that there are only two possible cases in the behaviour of $\mathfrak{h}_{p, N}(x), x \in[1 / 2,1]$. The first one concerns decreasing $\mathfrak{h}_{p, N}(x)$, its maximum is in $x=1 / 2$; the second one means increasing $\mathfrak{h}_{p, N}(x)$ to certain local maximum, and then it decreases, see the plots of $\mathfrak{h}_{2,2}(x), \mathfrak{h}_{27,2}(x)$ on Figures 1,2. Prove or disprove!

(3) To estimate the maximum value of $\mathfrak{h}_{p, N}(x)$ in the second case mentioned above.

(4) To obtain sharp estimates in Theorem 5. It has to be mentioned that for $p=2$ such sharp estimates were derived in [1].

(5) To apply the results obtained to the discontinuous signals, see [17].

(6) To apply the results obtained to the stochastic case, see [18].

\section{Acknowledgements}

The authors thank Professor Emeritus J.R. Higgins for numerous valuable comments, discussion and suggestions that have helped to improve our paper.

The recent investigation was supported in part by Research Project No. 1122352818-2814 of Ministry of Sciences, Education and Sports of Croatia and in part by La Trobe University Research Grant-501821 "Sampling, wavelets and optimal stochastic modelling".

\section{References}

[1] A.Ya. Olenko, T.K. Pogány, On sharp bounds for remainders in multidimensional sampling theorem, Sampl. Theory Signal Image Process. 6 (2007), No. 3, 249-272. 
[2] M. Plancherel, Gy. Pólya, Fonctions entières et intégrales de Fourier multiples, Comment. Math. Helv. 9 (1937), 224-248.

[3] M. Plancherel, Gy. Pólya, Fonctions entières et intégrales de Fourier multiples II, Comment. Math. Helv. 10 (1937), 110-163.

[4] R.P. Bots JR., Entire functions bounded on a line, Duke Math. J. 6 (1940,) 148-169.

[5] A.M. Lindner, On lower bounds of exponential frames, J. Fourier Anal. Appl. 5 (1999), No. 2, 187-194.

[6] S.M. NikolskiI , Approximation of Functions of Several Variables and Imbedding Theorems, Nauka, Moscow, 1969. (in Russian). English translation: Springer-Verlag, New York, 1975.

[7] H. TRIEBEL, Theory of Function spaces II, Birkhäuser Verlag, Basel, 1992.

[8] Y.-S. HAN, Plancherel-Pólya type inequality on spaces of homogeneous type and its applications, Proc. Amer. Math. Soc. 126 (1998), No. 11, 3315-3327.

[9] I. Pesenson, Plancherel-Pólya type inequalites for entire functions of exponential type in $L_{p}\left(\mathbb{R}^{d}\right)$, J. Math. Anal. Appl. 330 (2007), No. 2, 1194-1206.

[10] J.R. Higgins, Sampling in Fourier and Signal Analysis: Foundations, Clarendon Press, Oxford, 1996.

[11] Li Xin Min, Uniform bounds for sampling expansions, J. Approx. Theory 93(1998), No. $1,100-113$.

[12] A.YA. Olenko, T.K. PogÁnY, Sharp exact upper bound for interpolation remainder of random processes, Theor. Probab. Math. Statist. 71 (2004) 133-144.

[13] T.K. PogÁny, H.M. Srivastava and Ž. Tomovski, Some families of Mathieu a-series and alternating Mathieu a-series, Appl. Math. Comput. 173 (2006) 69-108.

[14] K.M. Flornes, Yu. LyubarskiI And K. SeIP, A direct interpolation method for irregular sampling, Appl. Comput. Harmon. Anal. 8 (2000), No. 1, 113-121.

[15] J.L. Yen, On nonuniform sampling of bandwidth limited signals, IRE Trans. Circuit Theory CT-3 (1956) 251-257.

[16] Jinfan LOng, Gensun FAnG, On uniform truncation error upper bounds and alisaing error for multidimensional sampling expansion, Sampl. Theory Signal Image Process. 2 ( 2003), No. 2, 103-115.

[17] C. Bardaro, P.L. Butzer, R.L. Stens and G. Vinti, Approximation error of the Whittaker cardinal series in terms of an averaged modulus of smoothness covering discontinuous signals, J. Math. Anal. Appl. 316 (2006) 269-306.

[18] A.Ya. Olenko, T.K. PogÁny, Direct Lagrange-Yen type interpolation of random fields, Theory Stoch. Process. 9(25) (2003), No. 3-4, 242-254.

Department of Mathematics and Statistics, La Trobe University, Victoria 3086, Australia

E-mail address: a.olenko@latrobe.edu.au

Faculty of Maritime Studies, University of Rijeka, 51000 Rijeka, Studentska 2, Croatia

E-mail address: poganj@brod.pfri.hr 